\title{
Duality in non-abelian algebra II. From Isbell bicategories to Grandis exact categories
}

\author{
Zurab Janelidze ${ }^{1}$ - Thomas Weighill ${ }^{1,2,3}$
}

Received: 6 March 2014 / Accepted: 17 June 2015 / Published online: 4 January 2016

(C) Tbilisi Centre for Mathematical Sciences 2015

\begin{abstract}
This paper continues the study of self-dual axioms on forms, i.e. faithful amnestic functors, motivated by properties of subobject forms in non-abelian algebra (which in many cases are Grothendieck bifibrations), and in particular, by properties of forms of substructures of group-like structures. In this paper we explore axiomatic origins of this kind, of a hierarchy of contexts introduced by M. Grandis for his projective approach to non-abelian homological algebra. This reveals new links between those contexts and the theory of factorization systems. Among other things, we show that a Grandis exact category is the same as an Isbell bicategory whose form (fibration) of projections is isomorphic to the form (opfibration) of injections.
\end{abstract}

Keywords Grothendieck bifibration · Factorization system · Ex4-category · Exact form · Universalizer

\section{Introduction}

The aim of this series of papers is to study duality phenomena in various parts of nonabelian algebra, which is revealed by replacing the standard context of a category, with

Communicated by Marino Gran.

Supported by the South African National Research Foundation (Z. Janelidze, T. Weighill) and the MIH Media Lab at Stellenbosch University (T. Weighill).

$凶$ Thomas Weighill

tweighil@vols.utk.edu

1 Mathematics Division, Department of Mathematical Sciences, Stellenbosch University, Private Bag X1, Matieland 7602, South Africa

2 MIH Media Lab, K402 Knowledge Centre, Private Bag X1, Matieland 7602, South Africa

3 Department of Mathematics, University of Tennessee, Knoxville, TN 37996, USA 
that of a category $\mathbb{C}$ equipped with a form $F$ over $\mathbb{C}$, i.e. a faithful amnestic functor $F$ to $\mathbb{C}$. In some sense, the context of a category equipped with a form is a suitable refinement of the context of a lattice-ordered bicategory introduced in [13] for a similar purpose, and which, while effective for "abelian algebra" (leading to the notion of an abelian category), turned out to be less effective for a self-dual treatment of topics from "non-abelian algebra", such as, say, isomorphism theorems and homological diagram lemmas for non-abelian groups-in spite of the attempt by Wyler [14]. Before we can begin to use forms in developing a self-dual treatment of such topics, we need to organize a suitable system of dual axioms on a form. These axioms will be found by establishing "classification theorems"- theorems which classify, via axioms on forms, different types of categories, or structures on categories, that are encountered in modern categorical algebra. Several such classification theorems were obtained in [8], which gave rise to this series of papers, as well as in the first paper [9] from the series. In the present paper, relying on results obtained in [9], we extend this collection of theorems with several new such theorems. These theorems identify dual axioms which are inherent in the exactness axioms considered by Grandis in [4-6]. Examples of forms which satisfy some or all of these new axioms include the forms of normal subobjects over various categories of algebras (see Sect. 7), as well as the form of subobjects over any normal category [10], e.g. the category of groups.

\section{Preliminaries}

We begin by recalling some basic notions and notation/terminology from [9], and introducing some new ones, required for the present paper. Consider a form $F: \mathbb{B} \rightarrow$ $\mathbb{C}$. As usual, the fibre of $F$ at an object $X$ in $\mathbb{C}$, written as $F^{-1}(X)$, is the ordered set (class) given by the subcategory of $\mathbb{B}$ consisting of those objects which by $F$ are mapped to $X$, and those morphisms which by $F$ are mapped to the identity morphism $1_{X}$. We write $1^{X}$ (and sometimes simply 1 ) for the upper bound of $F^{-1}(X)$, when it exists, and $0^{X}$ (or simply 0 ) for the lower bound, again when it exists. We say that a form is locally bounded if each of its fibres have both upper and lower bounds. When a morphism $f: X \rightarrow Y$ has a cocartesian lifting at an object $A \in F^{-1}(X)$, the codomain of this cocartesian lifting will be denoted by $f A$ (and sometimes by $f \cdot A$ ). Dually, when $f$ has a cartesian lifting at $B \in F^{-1}(Y)$, the domain of this lifting will be denoted by $B f$ (and sometimes by $B \cdot f$ ). When we say that $B f$ (or $f A$ ) is defined we are making a claim/assumption that the object which it is supposed to represent exists, i.e. the (co)cartesian lifting of $f$ at $B$ (at $A$ ) exists.

The following lemmas are consequences of standard properties of (co)cartesian liftings of morphisms:

Lemma 2.1 Consider two morphisms $f: X \rightarrow Y$ and $g: Y \rightarrow Z$, and an object $A \in F^{-1}(X)$. If $f \cdot A$ is defined, then $g \cdot(f \cdot A)$ is defined if and only if $(g \circ f) \cdot A$ is defined, and $g \cdot(f \cdot A)=(g \circ f) \cdot A$ when they are defined. Dually, for any object $B \in F^{-1}(Z)$, if $B \cdot g$ is defined then $(B \cdot g) \cdot f$ is defined if and only if $B \cdot(g \circ f)$ is defined, and $(B \cdot g) \cdot f=B \cdot(g \circ f)$ when they are defined. 
Lemma 2.2 Consider a morphism $f: X \rightarrow Y$ and two objects $A_{1}, A_{2} \in F^{-1}(X)$. If both $f \cdot A_{1}$ and $f \cdot A_{2}$ are defined, then $A_{1} \leqslant A_{2}$ implies $f \cdot A_{1} \leqslant f \cdot A_{2}$. Dually, for any two objects $B_{1}, B_{2} \in F^{-1}(Y)$, if both $B_{1} \cdot f$ and $B_{2} \cdot f$ are defined, then $B_{1} \leqslant B_{2}$ implies $B_{1} \cdot f \leqslant B_{2} \cdot f$.

Henceforth in this section we work in a category $\mathbb{C}$ equipped with a form $F$ which is bounded in the following sense:

Definition 2.3 A form $F$ over a category $\mathbb{C}$ is said to be bounded when it is locally bounded and for any morphism $f: X \rightarrow Y$ in $\mathbb{C}$, both $f \cdot 1^{X}$ and $0^{Y} \cdot f$ are defined.

In a bounded form $F$, a left universalizer of an object $B \in F^{-1}(Y)$ is a morphism $f: X \rightarrow Y$ which is terminal with the property that $f \cdot 1^{X} \leqslant B$, i.e. it has this property and for any other morphism $f^{\prime}: X^{\prime} \rightarrow Y$ having the same property $f^{\prime} \cdot 1^{X^{\prime}} \leqslant B$, there exists a unique morphism $x: X^{\prime} \rightarrow X$ such that $f^{\prime}=f x$. Similarly, a right universalizer of an object $A \in F^{-1}(X)$ is a morphism $f: X \rightarrow Y$ which is initial with the property that $A \leqslant 0^{Y} \cdot f$, i.e. it has this property and for any other morphism $f^{\prime}: X \rightarrow Y^{\prime}$ having the same property $A \leqslant 0^{Y^{\prime}} \cdot f^{\prime}$, there exists a unique morphism $y: Y \rightarrow Y^{\prime}$ such that $f^{\prime}=y f$.

The notion of a bounded form is self-dual, in the sense that a form $F: \mathbb{B} \rightarrow \mathbb{C}$ is bounded if and only if the dual form $F^{\mathrm{OP}}: \mathbb{B}^{\mathrm{OP}} \rightarrow \mathbb{C}^{\mathrm{OP}}$ is bounded. In a similar sense, the notion of a left universalizer is dual to the notion of a right universalizer: $f$ is a left universalizer of $B$, in a form $F: \mathbb{B} \rightarrow \mathbb{C}$, if and only if $f$ is a right universalizer of $B$ in the dual form $F^{\text {op }}: \mathbb{B}^{\text {op }} \rightarrow \mathbb{C}^{\text {op }}$.

Lemma 2.4 Any left universalizer $f$ of an object $B \in F^{-1}(Y)$ is necessarily a monomorphism, and $f^{\prime}$ is another left universalizer of the same object $B$ if and only if $f^{\prime}=f i$ for some isomorphism $i$. Dually, a right universalizer of an object $A \in F^{-1}(X)$ is an epimorphism, and if $f$ is a right universalizer of $A$ then $f^{\prime}$ is also a right universalizer of $A$ if and only if $f^{\prime}=j$ f for an isomorphism $j$.

We write lun $(B): \operatorname{Lun}(B) \rightarrow Y$ for any given left universalizer of $B \in F^{-1}(Y)$, when it exists, and we say that $\operatorname{lun}(B)$ is defined to claim/assume that $B$ has a left universalizer. Similarly, for right universalizers we use the notation $\operatorname{run}(A)$ for the morphism, and $\operatorname{Run}(A)$ for its codomain.

The proof of the above lemma uses the fact that if $f: X \rightarrow Y$ is an isomorphism then $f \cdot 1^{X}=1^{Y}$ (and dually, $0^{Y} \cdot f=0^{X}$ ). In general, a morphism $f: X \rightarrow Y$ such that $f \cdot 1^{X}=1^{Y}$ will be called a thick morphism. Dually, when $0^{Y} \cdot f=0^{X}$ we say that $f$ is thin.

A class $\mathcal{C}$ of morphisms is said to be a left class if it has the following properties:

- $\mathcal{C}$ contains all identity morphisms.

- $\mathcal{C}$ is closed under composition.

- If $f g \in \mathcal{C}$ then $g \in \mathcal{C}$.

Dually, $\mathcal{C}$ is said to be a right class if the first two conditions above hold, and $f g \in \mathcal{C}$ always implies $f \in \mathcal{C}$. Notice that the class of split monomorphisms is the smallest left class, and the class of split epimorphisms is the smallest right class. Note also that a left/right class always contains all isomorphisms. The class of all mono(/epi)morphisms is another example of a left(/right) class. 
Lemma 2.5 The class of all thin morphisms is a left class, and dually, the class of all thick morphisms is a right class.

An object $A \in F^{-1}(X)$ is said to be normal when $A=0^{Y} \cdot f$ for some morphism $f: X \rightarrow Y$ in $\mathbb{C}$. Dually, an object $B \in F^{-1}(Y)$ is said to be conormal when $B=f \cdot 1^{X}$ for some morphism $f: X \rightarrow Y$.

Lemma 2.6 Consider a left universalizer $f: X \rightarrow Y$ of an object $B \in F^{-1}(Y)$. Then:

- $f$ is also a left universalizer of $f \cdot 1^{X}$.

- $B$ is conormal if and only if $f \cdot 1^{X}=B$.

- $f$ is an isomorphism if and only if $B=1^{Y}$.

Dually, if $f$ is a right universalizer of an object $A \in F^{-1}(X)$, then $f$ is also a right universalizer of $0^{Y} \cdot f$ and $A$ is normal if and only if $0^{Y} \cdot f=A$, and finally, $f$ is an isomorphism if and only if $A=0^{X}$.

A morphism $f: X \rightarrow Y$ is said to be an embedding if for any two conormal objects $A_{1}, A_{2} \in F^{-1}(X)$ we have

$$
f \cdot A_{1} \leqslant f \cdot A_{2} \Rightarrow A_{1} \leqslant A_{2} .
$$

Dually, $f$ is a coembedding if for any two normal objects $B_{1}, B_{2} \in F^{-1}(Y)$ we have

$$
B_{1} \cdot f \leqslant B_{2} \cdot f \Rightarrow B_{1} \leqslant B_{2} .
$$

Lemma 2.7 The class of all (co)embeddings is a left (right) class.

\section{The general theory}

We recall from [3] (see also [1]) some notation and some very basic notions and results from the theory of factorization systems. A morphism $e$ is said to be orthogonal to a morphism $m$, written as $e \downarrow m$, if any commutative square of solid arrows

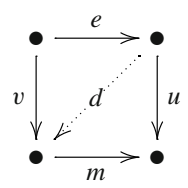

admits a unique diagonal fill-in $d$ which makes the two triangles inside the square commute. For two classes $\mathcal{E}$ and $\mathcal{M}$ of morphisms, we write $\mathcal{E} \downarrow \mathcal{M}$ when $e \downarrow m$ for all $e \in \mathcal{E}$ and $m \in \mathcal{M}$. Then, for a class $\mathcal{C}$ of morphisms,

$$
\mathcal{C}^{\downarrow}=\{m \mid \mathcal{C} \downarrow\{m\}\}, \quad \mathcal{C}^{\uparrow}=\{e \mid\{e\} \downarrow \mathcal{C}\} .
$$

A prefactorization system is a pair $(\mathcal{E}, \mathcal{M})$ such that $\mathcal{E}=\mathcal{M}^{\uparrow}$ and $\mathcal{E}^{\downarrow}=\mathcal{M}$. A factorization system is a prefactorization system $(\mathcal{E}, \mathcal{M})$ such that any morphism $f$ decomposes as $f=m e$ where $e \in \mathcal{E}$ and $m \in \mathcal{M}$. Already in a prefactorization 
system both classes $\mathcal{E}$ and $\mathcal{M}$ contain isomorphisms and are closed under composition. A factorization system can be equivalently defined as a pair $(\mathcal{E}, \mathcal{M})$ such that $\mathcal{E} \downarrow \mathcal{M}$ and in addition:

- both $\mathcal{E}$ and $\mathcal{M}$ contain identity morphisms and are closed under composition with isomorphisms;

- any morphism $f$ decomposes as $f=m e$ where $e \in \mathcal{E}$ and $m \in \mathcal{M}$.

Lemma 3.1 In a category equipped with a bounded form, we have $e \downarrow m$ in any of the following cases:

- when e is thick and $m$ is a left universalizer;

- when e is a right universalizer and $m$ is thin.

Proof Suppose $e$ is thick and $m$ is a left universalizer. Consider a commutative square (1) of solid arrows. Since $m$ is a monomorphism (Lemma 2.4), it suffices to show that $m d=u$ for some morphism $d$. Since $m$ is a left universalizer of $m \cdot 1$ (Lemma 2.6), the existence of such $d$ will follow from the inequality $u \cdot 1 \leqslant m \cdot 1$. Since $e$ is thick, we have: $u \cdot 1=u \cdot(e \cdot 1)=(u \circ e) \cdot 1=(m \circ v) \cdot 1=m \cdot(v \cdot 1)($ Lemma 2.1). At the same time, $m \cdot(v \cdot 1) \leqslant m \cdot 1$ (Lemma 2.2). Orthogonality of $e$ and $m$ in the case when $e$ is a right universalizer and $m$ is thin follows by duality.

Throughout the rest of this paper, for a bounded form $F$, we write $\mathcal{R}_{F}$ to denote the class of right universalizers and $\mathcal{L}_{F}$ to denote the class of left universalizers.

Definition 3.2 A bounded form $F$ over a category $\mathbb{C}$ is said to be

- a pre-exact form when every conormal object has a left universalizer and every normal object has a right universalizer;

- an orthogonal form when it is a pre-exact form and $\mathcal{R}_{F} \downarrow \mathcal{L}_{F}$;

- a closed orthogonal form when it is an orthogonal form and in addition $\mathcal{R}_{F}=\mathcal{R}_{F}^{\downarrow \uparrow}$ and $\mathcal{L}_{F}=\mathcal{L}_{F}^{\uparrow \downarrow}$

- an exact form when it is a pre-exact form and $\left(\mathcal{R}_{F}, \mathcal{L}_{F}\right)$ is a prefactorization system.

Proposition 3.3 For any pre-exactform $F$ we have: $\mathcal{L}_{F}^{\uparrow}$ is the class of thick morphisms and $\mathcal{R}_{F}^{\downarrow}$ is the class of thin morphisms.

Proof Already by Lemma 3.1, $\mathcal{L}_{F}^{\uparrow}$ contains all thick morphisms. If a morphism $f$ : $X \rightarrow Y$ belongs to $\mathcal{L}_{F}^{\uparrow}$, then we obtain a commutative diagram

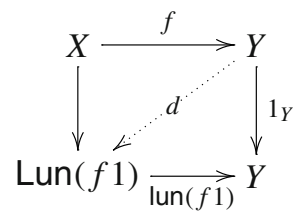

This gives that $\operatorname{lun}(f 1)$ is a split epimorphism. As lun $(f 1)$ is also a monomorphism (Lemma 2.4), we obtain that it is an isomorphism. Then $f 1=1$ (Lemma 2.6), showing that $f$ is thick. Dually, $\mathcal{R}_{F}^{\downarrow}$ is the class of thin morphisms. 
Note that as a consequence of the above proposition, we get that for a pre-exact form $F$ the following conditions are equivalent:

(i) $F$ is an orthogonal form.

(ii) Every left universalizer is thin.

(iii) Every right universalizer is thick.

We now characterize orthogonality of forms via existence of special factorizations of morphisms:

Theorem 3.4 For any bounded form $F$ over a category $\mathbb{C}$ the following conditions are equivalent:

(i) $F$ is an orthogonal form.

(ii) Each morphism $f: X \rightarrow Y$ in $\mathbb{C}$ admits a factorization $f=m \theta e$ where $m$ is a left universalizer of $f 1$ and $e$ is a right universalizer of $0 f$.

Proof (i) $\Rightarrow$ (ii): Suppose (i) holds. Consider any morphism $f: X \rightarrow Y$ in $\mathbb{C}$. Since $f 1$ is conormal, it has a left universalizer. Dually, $0 f$ has a right universalizer. We then obtain a commutative diagram, where the morphisms $u$ and $v$ arise from the universal properties of the given left and right universalizers, respectively:

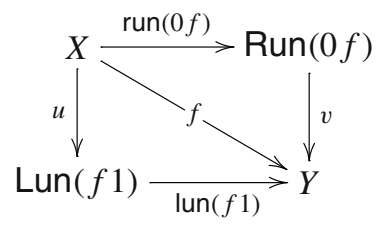

Now, orthogonality produces a diagonal fill-in:

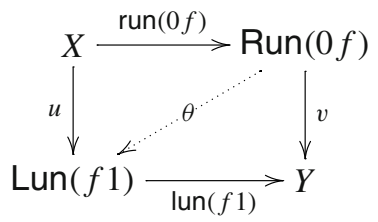

The zigzag in the above diagram is the desired factorization of $f$.

(ii) $\Rightarrow$ (i): Suppose that (ii) holds. For a conormal object $B=f \cdot 1^{X}$, the left universalizer of $B$ is the morphism $m$ in the factorization of $f$ given by (ii), which shows that every conormal object has a left universalizer. Dually, every normal object has a right universalizer. So $F$ is a pre-exact form. With Proposition 3.3 in mind, to show that $F$ is an orthogonal form, it suffices to show that any left universalizer is thin. If $f$ is a left universalizer, then $f$ is a left universalizer of $f 1$ (Lemma 2.6), which forces the composite $\theta e$ in the factorization $f=m \theta e$ given by (ii) to be an isomorphism (Lemma 2.4). This implies that $e$ is a split monomorphism. Since $e$ is a right universalizer, it is also an epimorphism (Lemma 2.4). Hence $e$ is an isomorphism. Now, $e$ is a right universalizer of $0 f$, and so $0 f=0$ (Lemma 2.6), showing that $f$ is thin. 
Closed orthogonal and exact forms can be also characterized via presence of suitable factorizations of morphisms, as the two theorems below show. In fact, as we will see, much more can be said in these two cases. We state both theorems before presenting their proofs:

Theorem 3.5 For any bounded form $F$ over a category $\mathbb{C}$ the following conditions are equivalent:

(i) $F$ is a closed orthogonal form.

(ii) Each morphism $f: X \rightarrow Y$ in $\mathbb{C}$ admits a factorization $f=m \theta e$ where $m$ is a thin left universalizer, $e$ is a thick right universalizer, and $\theta$ is both thick and thin.

(iii) Each morphism $f: X \rightarrow Y$ in $\mathbb{C}$ admits a factorization $f=m \theta e$ where $m$ is a left universalizer of $f 1$, $e$ is a right universalizer of $0 f$, and $\theta$ is both thick and thin.

(iv) $F$ is an orthogonal form with both $\mathcal{R}_{F}$ and $\mathcal{L}_{F}$ closed under composition.

(v) Every conormal object has a left universalizer which is a thin embedding, and dually, every normal object has right universalizer which is a thick coembedding.

(vi) $F$ is an orthogonal form and the pairs $\left(\mathcal{R}_{F}, \mathcal{R}_{F}^{\downarrow}\right)$ and $\left(\mathcal{L}_{F}^{\uparrow}, \mathcal{L}_{F}\right)$ are factorization systems.

Theorem 3.6 For any bounded form $F$ over a category $\mathbb{C}$ the following conditions are equivalent:

(i) $F$ is an exact form.

(ii) $F$ is a closed orthogonal form and any morphism in $\mathbb{C}$ that is both thick and thin is an isomorphism.

(iii) Each morphism $f: X \rightarrow Y$ in $\mathbb{C}$ admits a factorization $f=$ me where $m$ is a thin left universalizer, and e is a thick right universalizer.

(iv) Each morphism $f: X \rightarrow Y$ in $\mathbb{C}$ admits a factorization $f=$ me where $m$ is a left universalizer of $f 1$, and e is a right universalizer of $0 f$.

(v) $F$ is a pre-exact form and the pair $\left(\mathcal{R}_{F}, \mathcal{L}_{F}\right)$ is a factorization system.

Proof of Theorem 3.5 (i) $\Rightarrow$ (ii): Suppose $F$ is a closed orthogonal form. By Theorem 3.4 , we have a factorization

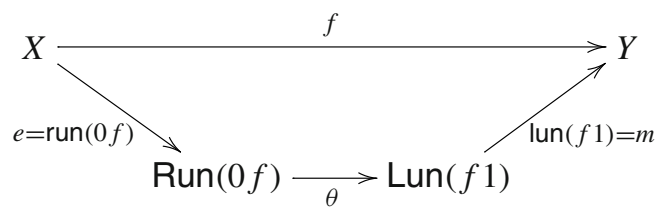

Since the form is orthogonal, $e$ is thick and $m$ is thin (Proposition 3.3). We would like to show that $\theta$ is both thick and thin. Since $\theta 1$ is conormal, it has a left universalizer. Since $\mathcal{L}_{F}=\mathcal{L}_{F}^{\uparrow \downarrow}$, a composite of two left universalizers is a left universalizer. In particular, the composite $m \circ \operatorname{lun}(\theta 1)$ is a left universalizer. Then, it is a left universalizer of $(m \circ \operatorname{lun}(\theta 1)) \cdot 1$ (Lemma 2.6). Since lun $(\theta 1) \cdot 1=\theta 1$ (Lemma 2.6), we have:

$$
(m \circ \operatorname{lun}(\theta 1)) \cdot 1=m \cdot(\operatorname{lun}(\theta 1) \cdot 1)=m \cdot \theta 1=(m \circ \theta) \cdot 1
$$


(Lemma 2.1). Now, since $e$ is thick, we further have:

$$
(m \circ \theta) \cdot 1=(m \circ \theta) \cdot(e 1)=(m \circ \theta \circ e) \cdot 1=f 1
$$

(Lemma 2.1). Thus, $m \circ \operatorname{lun}(\theta 1)$ is a left universalizer of $f 1$. But so is $m$, and hence $\operatorname{lun}(\theta 1)$ is an isomorphism (Lemma 2.4). Then, $\theta 1=1$ (Lemma 2.6). This shows that $\theta$ is thick. By a dual argument, $\theta$ is also thin.

(ii) $\Rightarrow$ (iii): Suppose (ii) holds. Consider a factorization

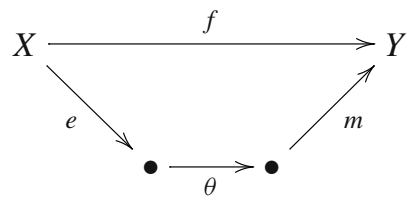

where $e$ is a thick right universalizer, $m$ is a thin left universalizer, and $\theta$ is both thick and thin. Since both $e$ and $\theta$ are thick, so is their composite $\theta e$ (Lemma 2.5). Then $m 1=m \cdot(\theta e \cdot 1)=(m \theta e) \cdot 1=f 1$ (Lemma 2.1). So $m$ is a left universalizer of $m 1=f 1$ (Lemma 2.6). Dually, $e$ is a right universalizer of $0 f$.

(iii) $\Rightarrow$ (iv): Suppose (iii) holds. Then $F$ is an orthogonal form by Theorem 3.4, and so by Proposition 3.3, every left universalizer is thin and every right universalizer is thick. We show that $\mathcal{L}_{F}$ is closed under composition. Consider a composite $m_{2} m_{1}$ of two left universalizers, and a commutative diagram of solid arrows

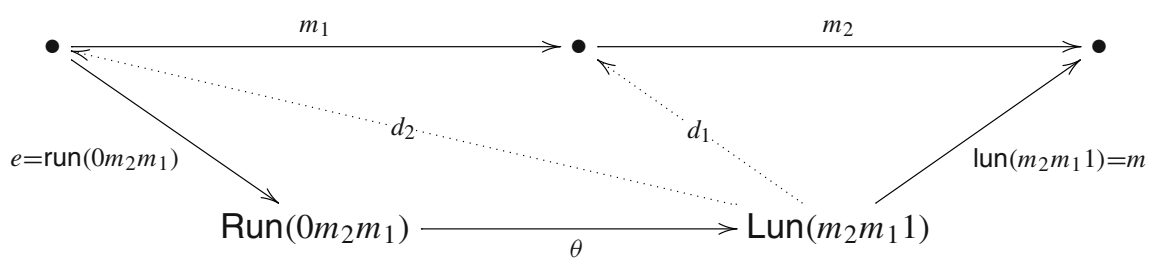

obtained by (iii). Since $e$ is thick and $\theta$ is thick, so is their composite $\theta e$ (Lemma 2.5). The orthogonality $\theta e \downarrow m_{2}$ (Lemma 3.1) produces a morphism $d_{1}$ such that $m_{2} d_{1}=m$ and $d_{1} \theta e=m_{1}$. Next, the orthogonality $\theta e \downarrow m_{1}$ produces a morphism $d_{2}$ such that $d_{2} \theta e$ is an identity morphism, and $m_{1} d_{2}=d_{1}$. Altogether, since the $m$ 's are monomorphisms (Lemma 2.4), we obtain that $\theta e$ is an isomorphism (with inverse $d_{2}$ ). Then, since $m_{2} m_{1}=m \theta e$ and $m$ is a left universalizer, $m_{2} m_{1}$ is also a left universalizer (Lemma 2.4). This shows that $\mathcal{L}_{F}$ is closed under composition. Dually, $\mathcal{R}_{F}$ is closed under composition.

(iv) $\Rightarrow(v)$ : Suppose that (iv) holds. Consider a left universalizer $f: X \rightarrow Y$ and two conormal objects $A_{1}, A_{2} \in F^{-1}(X)$. Then $A_{1}=\operatorname{lun}\left(A_{1}\right) \cdot 1$ and $A_{2}=\operatorname{lun}\left(A_{2}\right) \cdot 1$ (Lemma 2.6). Suppose $f \cdot A_{1} \leqslant f \cdot A_{2}$. By (iv), the composite $f$ lun( $\left(A_{2}\right)$ is a left universalizer, and hence it is a left universalizer of $\left(f \operatorname{lun}\left(A_{2}\right)\right) \cdot 1$ (Lemma 2.6). Now, $\left(f \operatorname{lun}\left(A_{2}\right)\right) \cdot 1=f \cdot\left(\operatorname{lun}\left(A_{2}\right) \cdot 1\right)=f \cdot A_{2}$ (Lemma 2.1), and similarly, $\left(f \operatorname{lun}\left(A_{1}\right)\right) \cdot 1=f \cdot A_{1}$. So, $f \cdot A_{1} \leqslant f \cdot A_{2}$ implies $f$ lun $\left(A_{1}\right)=f$ lun $\left(A_{2}\right) u$ for some morphism $u$. Since $f$ is a monomorphism (Lemma 2.4), we get $\operatorname{lun}\left(A_{1}\right)=\operatorname{lun}\left(A_{2}\right) u$. 
This in turn gives $A_{1}=\operatorname{lun}\left(A_{1}\right) \cdot 1=\left(\operatorname{lun}\left(A_{2}\right) u\right) \cdot 1=\operatorname{lun}\left(A_{2}\right) \cdot(u \cdot 1)(\operatorname{Lemma} 2.1)$. Finally, $\operatorname{lun}\left(A_{2}\right) \cdot(u \cdot 1) \leqslant \operatorname{lun}\left(A_{2}\right) \cdot 1=A_{2}$ (Lemma 2.2) and so $A_{1} \leqslant A_{2}$. This shows that any left universalizer is an embedding. Dually, any right universalizer is a coembedding. Since by (iv) the form is orthogonal, to obtain (v) it remains to apply Proposition 3.3.

$(v) \Rightarrow$ (vi): Suppose (v) holds. Then the form is pre-exact and so by Proposition 3.3, $\mathcal{L}_{F}^{\uparrow}$ is the class of thick morphisms and $\mathcal{R}_{F}^{\downarrow}$ is the class of thin morphisms. (v) implies that every left universalizer is thin, and so $\mathcal{R}_{F}^{\downarrow} \supseteq \mathcal{L}_{F}$ which is the same as $\mathcal{R}_{F} \downarrow$ $\mathcal{L}_{F}$. The form $F$ is therefore orthogonal. Consider a morphism $f: X \rightarrow Y$. By Theorem 3.4, it decomposes as $f=\operatorname{lun}(f 1) \circ \theta \circ \operatorname{run}(0 f)$. We claim that $\theta \circ \operatorname{run}(0 f)$ is thick. Indeed, on the one hand we have $f 1=\operatorname{lun}(f 1) \cdot 1$ (Lemma 2.6), and on the other hand, $f 1=(\operatorname{lun}(f 1) \circ \theta \circ \operatorname{run}(0 f)) \cdot 1=\operatorname{lun}(f 1) \cdot((\theta \circ \operatorname{run}(0 f)) \cdot 1)$ (Lemma 2.1). Since lun $(f 1)$ is an embedding, it follows that $1 \leqslant(\theta \circ \operatorname{run}(0 f)) \cdot 1$, and hence $1=(\theta \circ \operatorname{run}(0 f)) \cdot 1$, showing that $\theta \circ \operatorname{run}(0 f)$ is thick. So any morphism $f$ decomposes as a thick morphism followed by a left universalizer, which together with Lemma 3.1 and the fact that $\mathcal{L}_{F}^{\uparrow}$ and $\mathcal{L}_{F}$ contain identity morphisms and are closed under composition with isomorphisms (Lemmas 2.4 and 2.5), show that $\left(\mathcal{L}_{F}^{\uparrow}, \mathcal{L}_{F}\right)$ is a factorization system. Dually, $\left(\mathcal{R}_{F}, \mathcal{R}_{F}^{\downarrow}\right)$ is a factorization system.

(vi) $\Rightarrow$ (i): When $\left(\mathcal{L}_{F}^{\uparrow}, \mathcal{L}_{F}\right)$ and $\left(\mathcal{R}_{F}, \mathcal{R}_{F}^{\downarrow}\right)$ are factorization systems we have $\mathcal{L}_{F}=$ $\mathcal{L}_{F}^{\uparrow \downarrow}$ and $\mathcal{R}_{F}=\mathcal{R}_{F}^{\downarrow \uparrow}$.

Proof of Theorem 3.6 (i) $\Rightarrow$ (ii): Any exact form is a closed orthogonal form. When $F$ is pre-exact, $\mathcal{R}_{F}^{\downarrow} \cap \mathcal{L}_{F}^{\uparrow}$ is the class of morphisms which are both thick and thin (Proposition 3.3). When $\left(\mathcal{R}_{F}, \mathcal{L}_{F}\right)$ is a prefactorization system, $\mathcal{R}_{F}^{\downarrow} \cap \mathcal{L}_{F}^{\uparrow}=\mathcal{L}_{F} \cap \mathcal{R}_{F}$ is the class of isomorphisms.

(ii) $\Rightarrow$ (iii) follows from Theorem 3.5 .

(iii) $\Rightarrow$ (iv) by the same argument as the one used to prove (ii) $\Rightarrow$ (iii) in Theorem 3.5 , with $\theta$ there to be taken as an identity morphism.

(iv) $\Rightarrow$ (v): Suppose (iv) holds. By Theorem $3.5, F$ is a closed orthogonal form and so it is pre-exact and we have $\mathcal{L}_{F}=\mathcal{L}_{F}^{\uparrow \downarrow}$ and $\mathcal{R}_{F} \subseteq \mathcal{L}_{F}^{\uparrow}$. The existence of factorizations described in (iv) leaves to show that $\left(\mathcal{R}_{F}, \mathcal{L}_{F}\right)$ is a pre-factorization system, and for this it suffices to show that $\mathcal{L}_{F}^{\uparrow} \subseteq \mathcal{R}_{F}$. Recall from Proposition 3.3 that $\mathcal{L}_{F}^{\uparrow}$ is the class of thick morphisms. So we must show that any thick morphism is a right universalizer. If a morphism $f: X \rightarrow Y$ is thick, then since $f 1=1$ the morphism $m$ in the factorization $f=m e$ given by (iv) is an isomorphism (Lemma 2.6). Since in the same factorization $e$ is a right universalizer, we get that $f=m e$ is a right universalizer (Lemma 2.4).

$(v) \Rightarrow$ (i) since any factorization system is a prefactorization system.

\section{The binormal case}

As defined in [9], a form is binormal when in each fibre every object is both normal and conormal. In such a form, to say that every (co)normal object has a right (left) universalizer, is the same as to say that any object (in a fibre) has a right (left) univer- 
salizer. In this case we also say that the form admits right (left) universalizers. Notice that a binormal form is bounded if and only if it is locally bounded and is a bifibration.

According to Corollary 3.2 in [9], there is a bijection between the following structures on any given category $\mathbb{C}$ :

ideals $\mathcal{N}$ of null morphisms in $\mathbb{C}$
admitting kernels and cokernels $\quad \begin{gathered}\text { isomorphism classes of binormal } \\ \text { pre-exact forms } F \text { over } \mathbb{C}\end{gathered}$

Here, by an ideal $\mathcal{N}$ of null morphisms is meant a class $\mathcal{N}$ of morphisms which is closed under composition with morphisms in $\mathbb{C}$. This notion was first introduced and studied in [2,12] (and its additive version, independently in [11]), and gives the basis for the work of M. Grandis on categorical foundation of homological algebra (see [4-6]). Kernels and cokernels relative to an ideal are defined in a similar way as in the case of a pointed category (where the ideal has exactly one morphism from any object $X$ to any object $Y)$ :

- A kernel (or more elaborately, an $\mathcal{N}$-kernel) of a morphism $g: B \rightarrow C$ in $\mathbb{C}$ is a morphism $k: A \rightarrow B$ such that $g k \in \mathcal{N}$ and $k$ is terminal with this property, i.e. if $g k^{\prime} \in \mathcal{N}$ for some morphism $k^{\prime}: A^{\prime} \rightarrow B$ then $k^{\prime}=k u$ for a unique morphism $u: A^{\prime} \rightarrow A$.

- A cokernel (or more elaborately, an $\mathcal{N}$-cokernel) of a morphism $f: A \rightarrow B$ in $\mathbb{C}$ is a morphism $c: B \rightarrow C$ such that $c f \in \mathcal{N}$ and $c$ is initial with this property, i.e. if $c^{\prime} f \in \mathcal{N}$ for some morphism $c^{\prime}: B \rightarrow C^{\prime}$ then $c^{\prime}=u c$ for a unique morphism u $: C \rightarrow C^{\prime}$.

The bijection above is given by assigning to an isomorphism class of a binormal bounded form $F$ admitting left and right universalizers, the class $\mathcal{N}=F^{*}$ of $F$-trivial morphisms, i.e. those morphisms $n: X \rightarrow Y$ for which $n \cdot 1^{X}=0^{Y}$ (or, equivalently, $1^{X}=0^{Y} \cdot n$ ). It then turns out that a kernel $k$ of a morphism $f$ is the same as a left universalizer of $0 f$, and dually, a cokernel $c$ of $f$ is the same as a right universalizer of $f 1$. Moreover, since any object in a fibre is both normal and conormal, it follows that the class of left universalizers coincides with the class of kernels, and the class of right universalizers coincides with the class of cokernels.

In [9], the bijection (2) is obtained as a restriction of a bijection

$\begin{aligned} \text { ideals } \mathcal{N} \text { of null morphisms in } \mathbb{C} & \approx \begin{array}{c}\text { isomorphism classes of binormal } \\ \text { admitting "enough exact sequences" }\end{array}\end{aligned}$

defined in the same way as (2): it assigns to an isomorphism class of a binormal bounded form $F$ the class $\mathcal{N}=F^{*}$ of $F$-trivial morphisms. As remarked in [9], in this case still the class of left/right universilzers coincides with the class of $F^{*}$ kernels/-cokernels. The property of admitting "enough exact sequences" on an ideal in the bijection above asks that each morphism $g: B \rightarrow C$ is part of a sequence

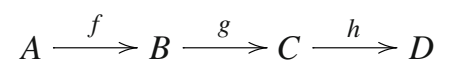


which is exact at $B$ and at $C$ (in the sense of [4]). Recall that exactness of the above sequence at, say $B$, means that $g f \in \mathcal{N}$ and for any two morphisms $f^{\prime}: A^{\prime} \rightarrow B$ and $g^{\prime}: B \rightarrow C^{\prime}$, if $g f^{\prime} \in \mathcal{N}$ and $g^{\prime} f \in \mathcal{N}$ then $g^{\prime} f^{\prime} \in \mathcal{N}$. For instance, the sequence above is exact as soon as $f$ is an $\mathcal{N}$-kernel and $h$ is an $\mathcal{N}$-cokernel of $g$.

Lemma 4.1 In a category $\mathbb{C}$ equipped with a binormal pre-exact form $F$, a morphism $f: X \rightarrow Y$ is thin if and only if it is $F^{*}$-mono in the sense of [6], i.e. $f$ has a kernel which belongs to $F^{*}$. Dually, $f$ is thick if and only if $f$ is $F^{*}$-epi in the sense of [6], i.e. $f$ has a cokernel which belongs to $F^{*}$.

Proof Suppose $f$ is thin. Then its kernel $k$ is a left universalizer of $0 f=0$. This implies that $k 1=0$, i.e. $k \in F^{*}$. Conversely, if $k \in F^{*}$ then, since $0 f$ is conormal (as $F$ is binormal), $0=k 1=0 f$ and so $f$ is thin.

Recall that a semiexact category or exl-category in the sense of Grandis $[4,6]$ is a pair $(\mathbb{C}, \mathcal{N})$ where $\mathbb{C}$ is a category, and $\mathcal{N}$ is an ideal of null morphisms in $\mathbb{C}$ admitting kernels and cokernels, such that $\mathcal{N}$ is a closed ideal, i.e. any morphism from $\mathcal{N}$ factors through an isomorphism which belongs to $\mathcal{N}$. By Theorem 1.5.4 in [6], this latter additional requirement is equivalent to every kernel being $\mathcal{N}$-mono, and is also equivalent to every cokernel being $\mathcal{N}$-epi. So in view of the above lemma, in our language this is stating that a binormal pre-exact form defines a closed ideal if and only if every left universalizer is thin, and if and only if every right universalizer is thick. Thanks to Proposition 3.3, we know that for a pre-exact form the last two conditions are equivalent to orthogonality, and so we obtain:

Theorem 4.2 A binormal bounded form $F$ over a category $\mathbb{C}$ is an orthogonal form if and only if the pair $\left(\mathbb{C}, F^{*}\right)$ is a Grandis semiexact category (which is the same as a Grandis exl-category).

After this theorem, the implication (i) $\Rightarrow$ (ii) of Theorem 3.4 in the binormal case becomes the basic known result that in a semiexact category any morphism has a normal factorization - see Section 1.5.5 in [6].

An ex2-category in the sense of Grandis $[4,6]$ is an ex1-category in which the class of kernels and the class of cokernels are both closed under composition. So, from Theorem 3.5 we reobtain the following result included in Corollary 3.2 of [9]:

Theorem 4.3 A binormal bounded form $F$ over a category $\mathbb{C}$ is a closed orthogonal form if and only if the pair $\left(\mathbb{C}, F^{*}\right)$ is a Grandis ex2-category.

The equivalences (iii) $\Leftrightarrow$ (iv) $\Leftrightarrow(\mathrm{v})$ of Theorem 3.5 are established in Section 2.1.3 of [6], in the context of semiexact categories.

An exact category or an ex4-category in the sense of Grandis [4,6] is a semiexact category in which any morphism $f$ factories as $f=m e$ where $m$ is a kernel of a cokernel of $f$ and $e$ is a cokernel of a kernel of $f$. In the language of the underlying binormal form, this is the same as to say that $m$ is a left universalizer of $f 1$ and $e$ is a right universalizer of $0 f$. So, Theorem 3.6 gives:

Theorem 4.4 A binormal bounded form $F$ over a category $\mathbb{C}$ is an exact form if and only if the pair $\left(\mathbb{C}, F^{*}\right)$ is a Grandis exact category (which is the same as a Grandis ex4-category). 
In the context of semiexact categories, the equivalence (iv) $\Leftrightarrow$ (ii) of Theorem 3.6 is made explicit in Section 2.2.6 of [6].

Thus we have the following hierarchy of bijections:

\begin{tabular}{|c|c|c|}
\hline $\begin{array}{l}\text { ideals of null morphisms } \\
\text { admitting enough exact sequences }\end{array}$ & $\approx$ & $\begin{array}{l}\text { isomorphism classes of } \\
\text { binormal bounded forms }\end{array}$ \\
\hline$U$ & & $u$ \\
\hline $\begin{array}{l}\text { ideals of null morphisms } \\
\text { admitting kernels and cokernels }\end{array}$ & $\approx$ & $\begin{array}{l}\text { isomorphism classes of } \\
\text { binormal pre-exact forms }\end{array}$ \\
\hline$u$ & & $u$ \\
\hline $\begin{array}{c}\text { ex1-category structures } \\
(=\text { semiexact category structures })\end{array}$ & $\approx$ & $\begin{array}{l}\text { isomorphism classes of } \\
\text { binormal orthogonal forms }\end{array}$ \\
\hline$U$ & & $u$ \\
\hline ex2-category structures & $\approx$ & $\begin{array}{l}\text { isomorphism classes of } \\
\text { binormal closed orthogonal forms }\end{array}$ \\
\hline$u$ & & $U$ \\
\hline $\begin{array}{c}\text { ex4-category structures } \\
\text { (= exact category structures) }\end{array}$ & $\approx$ & $\begin{array}{l}\text { isomorphism classes of } \\
\text { binormal exact forms }\end{array}$ \\
\hline
\end{tabular}

\section{Grandis exact categories via Isbell bicategories}

As we saw in Theorem 3.6, any exact form gives rise to a proper factorization system, i.e. a factorization system $(\mathcal{E}, \mathcal{M})$ where $\mathcal{E}$ is a class of epimorphisms and $\mathcal{M}$ is a class of monomorphisms [3]. We now answer the question: which proper factorizations systems arise from binormal exact forms?

Theorem 5.1 For any category $\mathbb{C}$ there is a bijection

proper factorization systems

$(\mathcal{E}, \mathcal{M})$ such that the form $\approx$ isomorphism classes of

of $\mathcal{E}$-quotients is isomorphic binormal exact forms $F$ over $\mathbb{C}$

to the form of $\mathcal{M}$-subobjects

given by assigning to an isomorphism class of a binormal exact form $F$ the factorization system $(\mathcal{E}, \mathcal{M})$ where $\mathcal{E}$ is the class of right universalizers for $F$ and $\mathcal{M}$ is the class of left universalizers for $F$.

Here, the form of $\mathcal{M}$-subobjects is the usual opfibration of $\mathcal{M}$-subobjects and the form of $\mathcal{E}$-quotients is the usual fibration of $\mathcal{E}$-quotients (see [9]). Since these two constructions are dual to each other, we only recall explicitly one of them. In the form $F$ of $\mathcal{M}$-subobjects, the fibre $F^{-1}(X)$ at an object $X$ is given by the ordered set (class) of $\mathcal{M}$-subobjects, and for any morphism $f: X \rightarrow Y$ and object $A \in F^{-1}(X)$, 
a subobject of $X$ represented say by a morphism $m \in \mathcal{M}$, the subobject $f \cdot A$ of $Y$ is the one given by the $\mathcal{M}$-part $m^{\prime}$ of the $(\mathcal{E}, \mathcal{M})$-factorization $f m=m^{\prime} e$ of $f$.

Proof It was shown in [9] that an isomorphism class of binormal exact forms is uniquely determined both by the class $\mathcal{M}$ of left universalizers and the class $\mathcal{E}$ of right universalizers for any (and hence all) forms in the class. In detail, such $F$ can be recovered back, up to an isomorphism of forms, as the form of $\mathcal{M}$-subobjects and at the same time, as the form of $\mathcal{E}$-quotients. Moreover, by Theorem 3.6 these classes $\mathcal{E}$ and $\mathcal{M}$ form a proper factorization system $(\mathcal{E}, \mathcal{M})$. Since the form of $\mathcal{M}$-subobjects and the form of $\mathcal{E}$-quotients are both isomorphic to $F$, they are also isomorphic to each other. To prove the theorem it remains to show that any proper factorization system $(\mathcal{E}, \mathcal{M})$ where the form of $\mathcal{E}$-quotients is isomorphic to the form of $\mathcal{M}$-subobjects, arises in this way from a binormal exact form $F$. So let $(\mathcal{E}, \mathcal{M})$ be such factorization system. Then, as shown in [9], $\mathcal{E}$ is the class of right universalizers for the form $F$ of $\mathcal{E}$-quotients, which is normal, locally bounded below (i.e. each fibre has a bottom element), is a fibration and admits right universalizers. Now, since the form of $\mathcal{E}$-quotients is isomorphic to the form of $\mathcal{M}$-subobjects, the same form $F$ is conormal, locally bounded above, is an opfibration and admits left universalizersthe class of left universalizers being the class $\mathcal{M}$. Thus $F$ is binormal, and exact by Theorem 3.6.

Recall that an Isbell bicategory [7] is a category equipped with a class $\mathcal{E}$ of morphisms called projections and a class $\mathcal{M}$ of morphisms called injections, such that the pair $(\mathcal{E}, \mathcal{M})$ is a proper factorization system on $\mathbb{C}$. The above theorem together with Theorem 4.4 lead to the following conclusion:

Corollary 5.2 A Grandis exact category is the same as an Isbell bicategory in which the form of $\mathcal{E}$-quotients is isomorphic to the form of $\mathcal{M}$-subobjects, where $\mathcal{E}$ is the class of projections and $\mathcal{M}$ is the class of injections of an Isbell bicategory (which become the classes of cokernels and kernels, respectively, of the Grandis exact category).

After this, one may ask: which proper factorization systems arise from exact forms that are not necessarily binormal? The answer is all, as we now show:

Proposition 5.3 For any proper factorization system $(\mathcal{E}, \mathcal{M})$ on a category $\mathbb{C}$ there exists an exact form $F$ over $\mathbb{C}$ such that $\mathcal{E}$ is the class of right universalizers and $\mathcal{M}$ is the class of left universalizers for $F$.

Proof First, we construct the domain $\mathbb{B}$ of $F$. Objects of $\mathbb{B}$ are triples $(X, E, M)$ where

- $X$ is an object of $\mathbb{C}$,

- $E$ is a class of morphisms of $\mathbb{C}$ having the following property: either $E=\varnothing$ or there exists a morphism $e \in \mathcal{E}$ (which we will call a generator of $E$ ) such that the domain of $e$ is $X$, and $E$ is the class $E=\langle e]$ of all composites $u e$ where $u$ is any morphism in $\mathbb{C}$,

- $M$ is a class of morphisms of $\mathbb{C}$ having the following property: either $M=\varnothing$ or there exists a morphism $m \in \mathcal{M}$ (which we will call a generator of $M$ ) such that 
the codomain of $m$ is $X$, and $M$ is the class $M=[m\rangle$ of all composites $m v$ where $v$ is any morphism in $\mathbb{C}$,

- Exactly one of $E$ and $M$ is the empty set.

A morphism $f:(X, E, M) \rightarrow\left(X^{\prime}, E^{\prime}, M^{\prime}\right)$ in $\mathbb{B}$ is a morphism $f: X \rightarrow X^{\prime}$ in $\mathbb{C}$ such that the following conditions hold:

- for any morphism $m \in M$ we have $f m \in M^{\prime}$,

- for any morphism $e \in E^{\prime}$ we have $e f \in E$,

- if $M=\varnothing$ and $E^{\prime}=\varnothing$ then $f \in M^{\prime}$ or $f \in E$.

It is a routine to verify that composition and identity morphisms can be defined in $\mathbb{B}$ via composition and identity morphisms in $\mathbb{C}$. Then, mapping a morphism $f$ : $(X, E, M) \rightarrow\left(X^{\prime}, E^{\prime}, M^{\prime}\right)$ to the morphism $f: X \rightarrow X^{\prime}$ defines a faithful functor $F: \mathbb{B} \rightarrow \mathbb{C}$. In fact, $F$ is even amnestic, and hence it is a form. In each fibre $F^{-1}(X)$, the top element $1^{X}$ is given by the triple $\left(X, \varnothing,\left[1_{X}\right\rangle\right)$, while the bottom element is given by the triple $\left(X,\left\langle 1_{X}\right], \varnothing\right)$. So, $F$ is locally bounded. It is not difficult to show that $F$ is in fact a bounded form where for each morphism $f: X \rightarrow Y$ we have $f \cdot 1^{X}=(Y, \varnothing,[m\rangle)$ and and $0^{Y} \cdot f=(X,\langle e], \varnothing)$ where $m$ and $e$ constitute an $(\mathcal{E}, \mathcal{M})$-factorization $f=m e$ of $f$. Then, it is again not difficult to show that $m$ is a left universalizer of $f \cdot 1^{X}$ and $e$ is a right universalizer of $0^{Y} \cdot f$, and hence $F$ is an exact form. We also get at once that every morphism in $\mathcal{M}$ is a left universalizer and every morphism in $\mathcal{E}$ is a right universalizer. Moreover, we get that $\mathcal{M}$ is the class of left universalizers of conormal objects and $\mathcal{E}$ is the class of right universalizers of normal objects. Via Lemma 2.6 this implies that $\mathcal{M}$ is the class of all left universalizers and $\mathcal{E}$ is the class of all right universalizers.

\section{Exactness up to a class of morphisms and Grandis ex3-categories}

In analogy with the terminology used in $[4,6]$, we call a morphism $f$ in a category equipped with a bounded form exact up to $\Theta$, where $\Theta$ is a class of morphisms in the category, if $f$ admits a factorization $f=m \theta e$ where $m$ is a left universalizer of $f 1$, $e$ is a right universalizer of $0 f$, and $\theta \in \Theta$.

By Theorems 3.4, 3.5 and 3.6, a bounded form over a category is

- orthogonal, if and only if any morphism in the category is exact up to the class of all morphisms;

- closed orthogonal, if and only if any morphism in the category is exact up to the class of morphisms which are both thick and thin;

- exact, if and only if any morphism in the category is exact up to the class of identity morphisms, or equivalently, up to the class of isomorphisms.

In view of the fourth bijection in the table (3), the ex3-categories or homological categories in the sense of Grandis $[4,6]$ are precisely those categories equipped with a binormal closed orthogonal form in which any morphism $f$ which decomposes as $f=e m$ where $m$ is a left universalizer and $e$ is a right universalizer such that $0 e \leqslant m 1$, is exact up to the class of identity morphisms. 


\section{Some examples}

We begin by recalling some examples from [6]. The forms of normal subobjects over the following pointed categories are binormal and orthogonal, but not closed orthogonal:

- the category of groups, where normal subobjects are given by normal subgroups;

- the category of rings without unit, where normal subobjects are given by ideals.

The forms of normal subobjects in the following pointed categories are binormal closed orthogonal, with the corresponding ex2-category being homological, but not exact:

- the category of lattices and Galois connections, where normal subobjects are given by principal down-closed sets;

- the category of commutative monoids, where normal subobjects are given by submonoids $H$ that satisfy $[a+h \in H \wedge h \in H] \Rightarrow[a \in H]$;

- the category of topological vector spaces (over a given topological field), where normal subobjects are given by closed linear subspaces;

- the category of Banach spaces (over the field of reals or complex numbers) and bounded linear mappings, where normal subobjects are given by closed linear subspaces;

- the category of pointed sets, where normal subobjects are given by subsets containing the base point.

In any abelian category, the form of normal subobjects (which are the same as subobjects) is binormal exact. The form of subobjects in any normal category in the sense of [10] is exact, but in general is not binormal; in fact, to ask binormality would be equivalent to ask that the normal category is abelian. A principal example of a nonabelian normal category is the category of groups (where subobjects are given by subgroups). In these cases, any object in a fibre is still conormal. A natural example of an exact form where this is no longer the case is the form of additive subgroups of unitary rings, over the category of unitary rings. In this form, for a unitary ring $R$, the fibre $F^{-1}(R)$ consists of additive subgroups of $R$, and a left/right action by a ring homomorphism is given by taking the inverse/direct image of an additive subgroup along the homomorphism. Then, conormal objects are subrings, while normal objects are ideals. But in fact, non-binormal examples of exact forms abound, as witnessed by Proposition 5.3.

We conclude by exploring a class of examples of forms, where bounded forms are automatically binormal and closed orthogonal forms, and a form is exact only in the trivial cases.

Consider an ordered set $O=(O, \leqslant)$. By an interval in $O$ we mean a nonempty subset $I$ of $O$ which satisfies one of the following conditions:

- There exists $a \in O$ such that $I=\{x \in O \mid a \leqslant x\}$.

- There exist $a, b \in O$ such that $I=\{x \in O \mid a \leqslant x \leqslant b\}$.

- There exists $b \in O$ such that $I=\{x \in O \mid x \leqslant b\}$.

Given two intervals $I$ and $J$ we write $I \leqslant J$ when for any $x \in I$ there exists $y \in J$ such that $x \leqslant y$, and at the same time, for any $y \in J$ there exists $x \in I$ such that 
$x \leqslant y$. It is easy to verify that this gives an order relation on the set of all intervals in $O$. We denote this ordered set, regarded as a category, by $O^{\prime}$. Next we define a form $F$ over $O^{\prime}$. The domain of $F$ is also an ordered set. Its objects are pairs $(I, x)$ where $I \in O^{\prime}$ and $x \in I$, where $(I, x) \leqslant(J, y)$ when $I \leqslant J$ and $x \leqslant y$. Then, $F$ maps a pair $(I, x)$ to its first component $I$. We call $F$ the form of intervals of $O$. Thus, in this form, the fibre $F^{-1}(I)$ at an interval $I$ is the interval $I$ with the order induced from $O$.

Theorem 7.1 (i) The form of intervals of an ordered set $O$ is locally bounded if and only if each connected component of $O$ has top and bottom elements.

(ii) The form of intervals of an ordered set $O$ is bounded if and only if each connected component of $O$ is a bounded lattice. Such form is always a binormal closed orthogonal form, and moreover, the corresponding Grandis ex2-category is homological.

(iii) The form of intervals of an ordered set $O$ is bounded and has every morphism (in the codomain of the form) exact up to the class of those morphisms which are at the same time embeddings and coembeddings, if and only if each connected component of $O$ is a bounded modular lattice.

(iv) The form of intervals of an ordered set $O$ is bounded and has every nontrivial morphism (in the codomain of the form) exact up to the class of identity morphisms, if and only if each connected component of $O$ is a bounded linearly ordered set.

(v) The form of intervals of an ordered set $O$ is exact if and only if $O$ is discrete, i.e. each connected component of $O$ is a singleton.

Proof (i) Local boundedness means that each interval has top and bottom elements. Any interval in $O$ lies in one of its connected components, and so if that connected component $C$ has top and bottom elements, then so will the interval. To prove the converse, consider a connected component $C$ and an element $a$ in $C$. We claim that the top element $u$ of the interval $\{x \mid a \leqslant x\}$ is the top element of $C$. Take any other element $b$ in $C$. Then we can form a zigzag in $C$ that connects $a$ and $b$, which can be displayed using a Hasse diagram as follows:
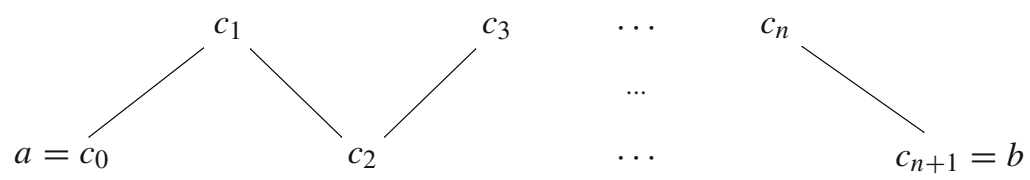

Notice that the top element in each interval $\left\{x \mid c_{i} \leqslant x\right\}$ must be the same as the top element of the interval $\left\{x \mid c_{i+1} \leqslant x\right\}$. This will yield $b \leqslant u$, proving that $u$ is the top element in $C$. Dually, each $C$ has a lower bound.

(ii) Suppose the form $F$ of intervals of an ordered set $O$ is bounded. Then, $F$ is locally bounded and so by (i), each connected component $C$ of $O$ has top and bottom elements. Let us denote them by 1 and 0 , respectively. Let $a, b \in C$. Consider the morphism

$$
f:[0, a] \rightarrow[b, 1]
$$


in the codomain of $F$, where $[i, j]$ denotes the interval $[i, j]=\{x \mid i \leqslant x \leqslant j\}$. By the definition of the element $f \cdot 1^{[0, a]}=f \cdot a$, this element is smallest among those elements $c \in[b, 1]$ for which $a \leqslant c$. Since $[b, 1]$ contains all elements of $O$ which are greater or equal to $b$, we conclude $f \cdot a=a \vee b$. Dually, $b \cdot f=a \wedge b$. This shows that $C$ is a bounded lattice. Conversely, suppose each connected component $C$ of $O$ is a bounded lattice. Then it is easy to see that each interval $I$ has the form $I=[i, j]$ for some $i, j, \in O$. Now, for a morphism

$$
f:[i, j] \rightarrow\left[i^{\prime}, j^{\prime}\right]
$$

in the codomain of $F$, it is a simple routine to verify that $j \vee i^{\prime}=f \cdot j$ and $j \wedge i^{\prime}=i^{\prime} \cdot f$. Notice that the required meets and joins exist since the presence of the morphism $f$ above forces $j$ and $i^{\prime}$ to be in the same connected component. Thus, $F$ is bounded.

Now, assuming that each connected component $O$ is a bounded lattice, we show that the form of intervals of $O$ is a binormal closed orthogonal form, and the corresponding Grandis ex2-category is homological. Consider an element $x \in[a, b]$. For the morphism $f:[a, x] \rightarrow[a, b]$, we have $f \cdot x=x \vee a=x$ which shows that $x$ is conormal. Dually, for the morphism $g:[a, b] \rightarrow[x, b]$, we have $x \cdot g=b \wedge x=x$ which shows that $x$ is normal. So the form $F$ is binormal. It is easy to see that the $f$ and the $g$ we obtained from $x$ are in fact, respectively, the left and the right universalizers of $x$. This makes it obvious that the left universalizers are thin embeddings, and the right universalizers are thick coembeddings. So $F$ is a binormal closed orthogonal form, thanks to Theorem 3.5. It is easy to verify that the corresponding Grandis ex2-category is homological.

(iii) Suppose the form of intervals of an ordered set $O$ is bounded, or equivalently, each connected component of $O$ is a bounded lattice. The decomposition of a morphism $f:[i, j] \rightarrow\left[i^{\prime}, j^{\prime}\right]$ as $f=m \theta e$ where $e$ is a right universalizer of $0 f$ and $m$ is a left universalizer of $f 1$ is given as follows:

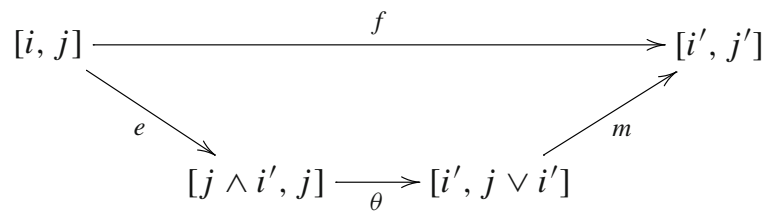

Left and right action by $\theta$ is defined as follows: $\theta \cdot x=x \vee i^{\prime}$ and $y \cdot \theta=j \wedge y$. To require that $\theta$ is an embedding and a coembedding is the same as to require that the Galois connection induced by this left and right action is an isomorphism $\left[j \wedge i^{\prime}, j\right] \approx\left[i^{\prime}, j \vee\right.$ $\left.i^{\prime}\right]$. The requirement that these isomorphisms hold in a given connected component $C$ of $O$ is precisely the requirement that the "diamond isomorphism theorem" holds in $C$, which is well known to be equivalent to modularity of $C$.

(iv) In the above setting, $f$ is trivial if and only if $j \vee i^{\prime}=i^{\prime}$, i.e. if and only if $j \leqslant i^{\prime}$. On the other hand, to ask that $\theta$ is an identity morphism is to ask that $i^{\prime} \leqslant j$. Thus, saying that any nontrivial morphism is exact up to the class of identity morphisms is equivalent to saying that in each connected component, whenever $j \leqslant i^{\prime}$ fails, $i^{\prime} \leqslant j$ 
must hold. Notice that we always have a canonical choice of $i$ and $j^{\prime}$, for a given $i^{\prime}$ and $j$-for instance, take $i$ to be the bottom element of the given connected component, and take $j^{\prime}$ to be the its biggest element, both of which exist due to (ii).

(v) Again in the setting of (iii), exactness asks that each $\theta$ is an isomorphism, or equivalently, an identity morphism (note that any isomorphism in the codomain of $F$ is an identity morphism), and so it asks that for any two elements $j$ and $i^{\prime}$ in the same connected component of $O$, we have $\left[j \wedge i^{\prime}, j\right]=\left[i^{\prime}, j \vee i^{\prime}\right]$ (since, similarly as before, we can always find appropriate $i$ and $j^{\prime}$ for a given $i^{\prime}$ and $j$ ). This is equivalent to each connected component being a singleton.

\section{References}

1. Carboni, A., Janelidze, G., Kelly, G.M., Paré, R.: On localization and stabilization for factorization systems. Appl. Categ. Struct. 5, 1-58 (1997)

2. Ehresmann, C.: Sur une notion générale de cohomologie. C. R. Acad. Sci. Paris 259, 2050-2053 (1964)

3. Freyd, P.J., Kelly, G.M.: Categories of continuous functors I. J. Pure Appl. Algebra 2, 169-191 (1972)

4. Grandis, M.: On the categorical foundations of homological and homotopical algebra. Cah. Topol. Géom. Diff. Catég. 33, 135-175 (1992)

5. Grandis, M.: Homological Algebra: The Interplay of Homology with Distributive Lattices and Orthodox Semigroups. World Scientific Publishing Co., Singapore (2012)

6. Grandis, M.: Homological Algebra In Strongly Non-Abelian Settings. World Scientific Publishing Co., Singapore (2013)

7. Isbell, J.R.: Algebras of uniformly continuous functions. Ann. Math. 68(2), 96-125 (1958)

8. Janelidze, Z.: On the form of subobjects in semi-abelian and regular protomodular categories. Appl. Categ. Struct. 22, 755-766 (2014)

9. Janelidze, Z., Weighill, T.: Duality in non-abelian algebra I. From cover relations to Grandis ex2-categories. Theory Appl. Categ. 29, 315-331 (2014)

10. Janelidze, Z.: The pointed subobject functor, $3 \times 3$ lemmas, and subtractivity of spans. Theory Appl. Categ. 23, 221-242 (2010)

11. Kelly, G.M.: Complete functors in homology. I. Chain maps and endomorphisms. In: Proceedings of Cambridge Philosophical Society 60, pp. 721-735 (1964)

12. Lavendhomme, R.: La notion d'idéal dans la théorie des catégories. Ann. Soc. Sci. Bruxelles Sér. 1(79), 5-25 (1965)

13. Mac Lane, S.: Duality for groups. Bull. Am. Math. Soc. 56, 485-516 (1950)

14. Wyler, O.: Weakly exact categories. Arch. Math. (Basel) 17, 9-19 (1966) 